\title{
APPLICATION POSSIBILITY OF SMARTPHONE AS PAYLOAD FOR PHOTOGRAMMETRIC UAV SYSTEM
}

\author{
MyungHyun Yun ${ }^{\text {a }}$, Jinsoo Kim ${ }^{\text {b }}$, Dongju Seo ${ }^{c}$, Jongchool Lee ${ }^{\mathrm{d}}$, Chuluong Choi ${ }^{\mathrm{a}, *}$ \\ a Department of Spatial Information Engineering, Pukyong National University, 599-1 Daeyeon3-Dong, Nam-Gu, \\ Busan 608-737, South Korea, Tel : +82-51-629-6655, Fax : +82-51-629-6653 \\ b ZEN21, 2nd Floor, RNC Building, 981-1 Bangbae 3-dong, Seocho-gu, Seoul , 137-848, South Korea \\ c, d Department of Civil Engineering, Pukyong National University, 365, Sinseon-ro, Nam-Gu, Busan, 608-739, South Korea
}

\section{Commission IV, WG IV/8}

KEY WORDS: UAV system, smartphone, android,

\begin{abstract}
:
Smartphone can not only be operated under 3G network environment anytime and anyplace but also cost less than the existing photogrammetric UAV since it provides high-resolution image, 3D location and attitude data on a real-time basis from a variety of built-in sensors. This study is aimed to assess the possibility of smartphone as a payload for photogrammetric UAV system. Prior to such assessment, a smartphone-based photogrammetric UAV system application was developed, through which real-time image, location and attitude data was obtained using smartphone under both static and dynamic conditions. Subsequently the accuracy assessment on the location and attitude data obtained and sent by this system was conducted. The smartphone images were converted into ortho-images through image triangulation. The image triangulation was conducted in accordance with presence or absence of consideration of the interior orientation (IO) parameters determined by camera calibration. In case IO parameters were taken into account in the static experiment, the results from triangulation for any smartphone type were within 1.5 pixel (RMSE), which was improved at least by $35 \%$ compared to when IO parameters were not taken into account. On the contrary, the improvement effect of considering IO parameters on accuracy in triangulation for smartphone images in dynamic experiment was not significant compared to the static experiment. It was due to the significant impact of vibration and sudden attitude change of UAV on the actuator for automatic focus control within the camera built in smartphone under the dynamic condition. This cause appears to have a negative impact on the image-based DEM generation. Considering these study findings, it is suggested that smartphone is very feasible as a payload for UAV system. It is also expected that smartphone may be loaded onto existing UAV playing direct or indirect roles significantly.
\end{abstract}

\section{INTRODUCTION}

Recently, Photogrammetric unmanned aerial vehicle (UAV) system is not only used to traditional monitoring fields such as natural disasters (e.g. landslides, wildfires) but also broadcasting. The system is consists of a digital camera, sensors (e.g. gyroscope, global positioning system) and communication devices (e.g. Wi-Fi, radio modem).

Since the experiment in which UAV was used for photogrammetric application for the first time (Przybilla and Wester-Ebbinghaus, 1979), many interesting studies have been conducted using various platforms (Bogacki et al., 2008; Fotinopoulos, 2004; Mihajlovic et al., 2008; Remondino et al., 2009). In UAV system the communications suite allows the operator to supervise and control the flying vehicle during the mission. Wzorek and others (2006) proposed the global system for mobile communications (GSM) technology as a communication media for an autonomous UAV.

However, UAV systems have payload limitation requires the use of light-weighted sensors, which often affect the accuracy of data provided by the small sensors.
On the other hand, state-of-the-art smartphones are lightweight in spite of having a variety of sensors (e.g. gyroscope, global position system (GPS), proximity and magnetometer). Therefore, the use of smartphone allows the system to be established at much lower cost than the existing UAV system. However, there has been no study on this using smartphone technology.

This study is aimed to assess the feasibility of smartphone as a payload for photogrammetric UAV system. Also, this paper is intended to carry out the development of the photogrammetric UAV system application to send and monitor the images automatically taken by the smartphone loaded onto UAV and the data obtained from a variety of sensors built in the smartphone.

\section{METHOD}

\subsection{Smartphone-based photogrammetric UAV system}

We developed the system that consists of UAV photography system, field monitoring system and UAV monitoring management system (Figure 1). UAV photography system consists of fixed-wing UAV (Multiplex Cularis electric glider

\footnotetext{
* Corresponding author.
} 
ARF 102.75"), Android OS-based smartphone loaded to UAV (one Samsung Galaxy S and two S2s) and bluetooth GPS (Ascen GPS 742). Galaxy S and S2 use Android OS 2.3 Gingerbread, which is a full touch bar type of smartphone (table 1). And the field monitoring system consists of a smartphone and a laptop computer to monitor various information (e.g. taken image, 3d location, attitude) obtained by smartphone in UAV photography system. Also, the UAV monitoring management system consists of a web server and a database server.

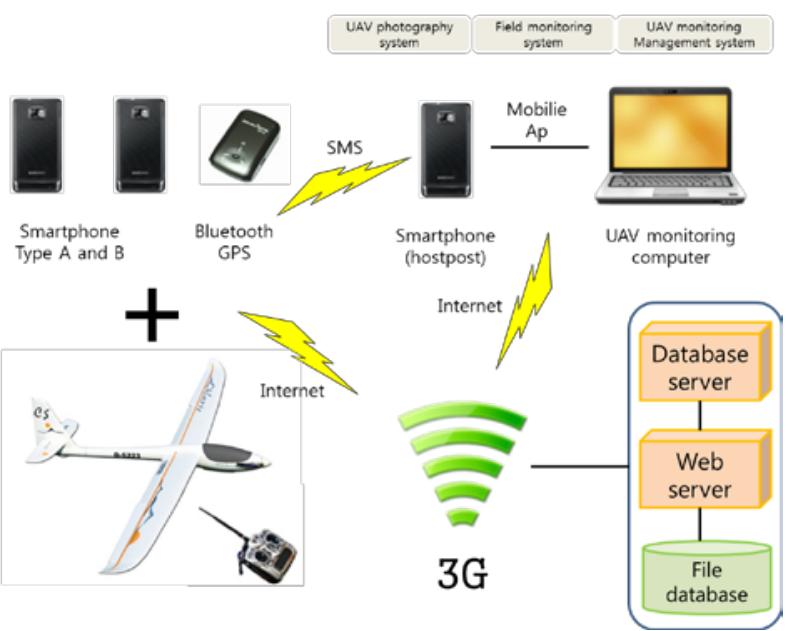

Figure 1. Diagram of the smartphone-based photogrammetric UAV system.

Table 1. Technical specification of Samsung Galaxy S and S2 (Samsung mobile, 2012a, 2012b)

\begin{tabular}{|c|c|c|}
\hline & Galaxy S & Galaxy S2 \\
\hline Device type & \multicolumn{2}{|c|}{ Smart phone (Full touch bar) } \\
\hline Operation System & \multicolumn{2}{|l|}{ Android 2.3 Gingerbread } \\
\hline Dimensions & $\begin{array}{l}122.4 \mathrm{~mm}(\mathrm{~L}) \times 64.2 \\
\mathrm{~mm}(\mathrm{~W}) \times 9.9 \mathrm{~mm}(\mathrm{H})\end{array}$ & $\begin{array}{l}125.3 \mathrm{~mm}(\mathrm{~L}) \text { x } 66.1 \\
\mathrm{~mm}(\mathrm{~W}) \times 8.49 \mathrm{~mm}(\mathrm{H})\end{array}$ \\
\hline Weight & $119 \mathrm{~g}$ & $116 \mathrm{~g}$ \\
\hline Talk time (3G) & about $393 \mathrm{~min}$ & about 9 hours \\
\hline Processor & Single core, $1000 \mathrm{MHz}$ & Dual core, $1200 \mathrm{MHz}$ \\
\hline Built-in storage & $16 \mathrm{~GB}$ & $32 \mathrm{~GB}$ \\
\hline Storage expansion & \multicolumn{2}{|c|}{ microSD, microSDHC (up to 32GB) } \\
\hline Display technology & Super AMOLED & Super AMOLED Plus \\
\hline Display physical size & 4.0 inches & 4.27 inches \\
\hline Display resolution & \multicolumn{2}{|l|}{$480 \times 800$ pixels } \\
\hline Battery capacity & $1500 \mathrm{mAh}$ & $1650 \mathrm{mAh}$ \\
\hline Camera imagesensor & \multicolumn{2}{|l|}{ CMOS (with Auto Focus) } \\
\hline Camera resolution & 5 megapixels & 9 megapixels \\
\hline Communication devices & \multicolumn{2}{|c|}{ WCDMA/GSM, Bluetooth, Wi-Fi } \\
\hline Sensors & $\begin{array}{l}\text { Accelerometer, } \\
\text { Magnetometer, } \\
\text { Proximity }\end{array}$ & $\begin{array}{l}\text { Accelerometer, } \\
\text { Magnetometer, } \\
\text { Proximity, Gyroscope }\end{array}$ \\
\hline
\end{tabular}

\subsection{Dynamic experiment}

The area for the dynamic experiment using UAV was located in Yangsan, Gyeongsangnam-do, in which a new town project is under construction (approximately 40,000 $\mathrm{m}^{2}$ ) (Figure 2a). There are gravels and sands for construction purpose ubiquitous all around the experimental area. In other words, since the experimental area contains various topographical relieves, it is appropriate to assess DEM generated using the smartphone images obtained through the developed system. Before actual flight, the survey to determine the coordinates of GCPs was conducted as in the static experiment, where the 2 criteria points were determined by GPS and 67 GCPs were determined by the total station (Topcon GPS-7001i) (Figure 2b). Since it is difficult to extract the feature point from the images taken in the field under construction, this study fabricated and use an aerial signal target on its own. In addition, terrestrial laser scanning was conducted to generate DEM for the test area and the obtained smartphone image processing was done by LPS.

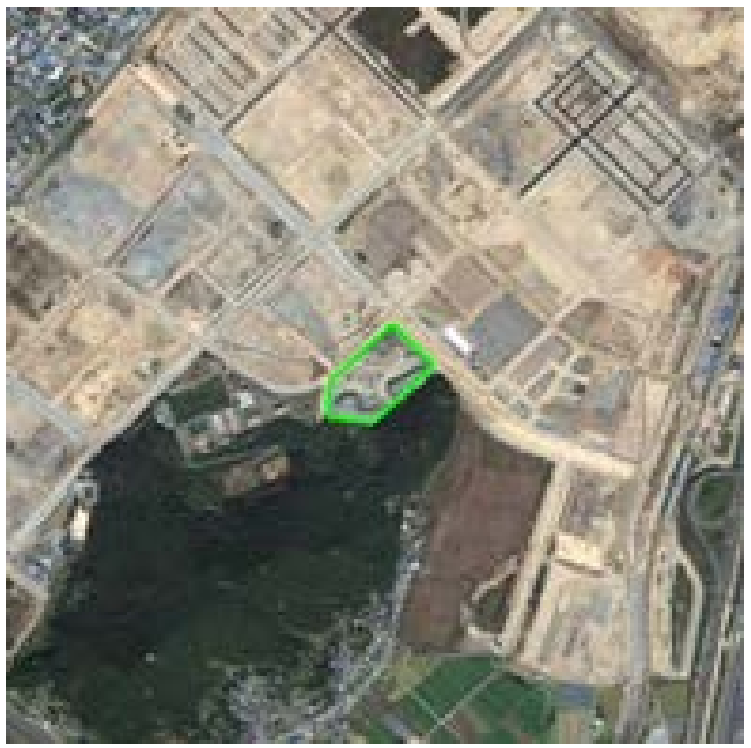

(a)

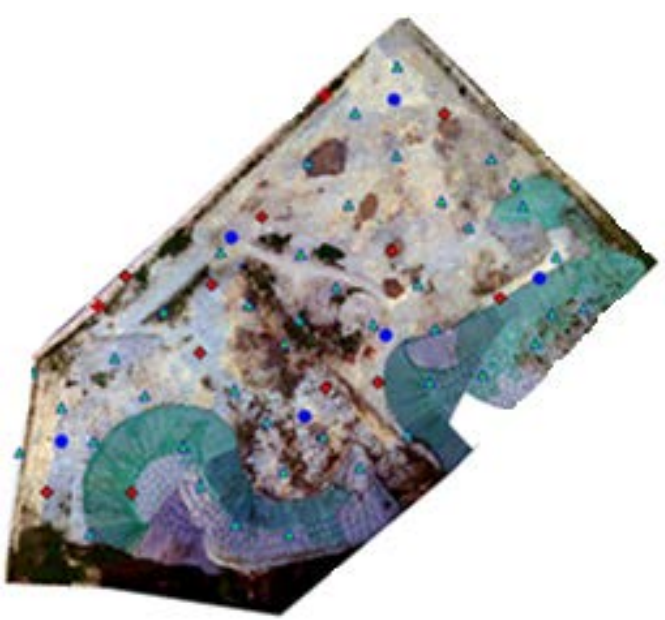

(b)

Figure 2. The experiment was conducted in the area of a new town project, with a very low likelihood of UAV crash or other such accidents. (a) Aerial view of the region in which the UAV images were taken. The central area (marked in green) is where the experiment was conducted. (b) Distribution of the locations of GPS base stations, 3D laser scanner, and GCPs on the final ortho-images of the experimental area. 


\section{RESULTS AND DISCUSSION}

\subsection{Performance of smartphone-based photogrammetric UAV system}

The automatic image-taking application is operated by Android OS-based smartphone. It was developed using Android 2.3 SDK. This consisted of the beginning and end of automatic image-taking, calculation of image-taking interval, environment configuration, smartphone camera test and link test with bluetooth GPS (Figure 3). Environment configuration provides the screen to manage UAV name, camera option, automatic image-taking option and bluetooth GPS setting. The configuration of focus mode and white balance is performed in the camera option within environment configuration, and the location of image-taking and effective radius are set in the automatic image-taking option.

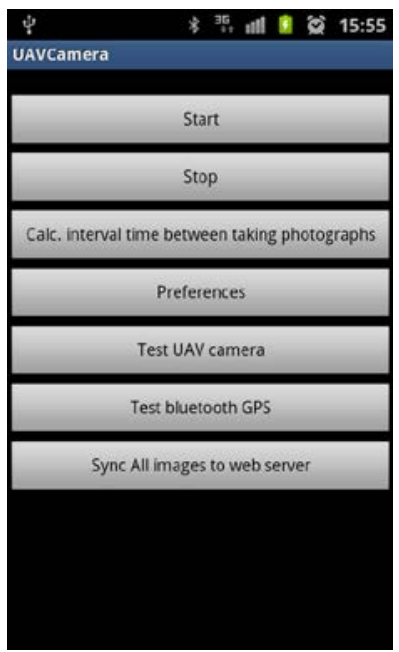

Figure 3. Screen for the implemented UAV photography system and field monitoring system application

We fabricate vibration-proof devices because in case ordinary gimbal device is attached to the fixed wing platform, it is likely to do serious damage to the attached devices when UAV makes landing The frame for this device was designed to be attached directly to the platform using very light-weighted balsa (Figure 4).

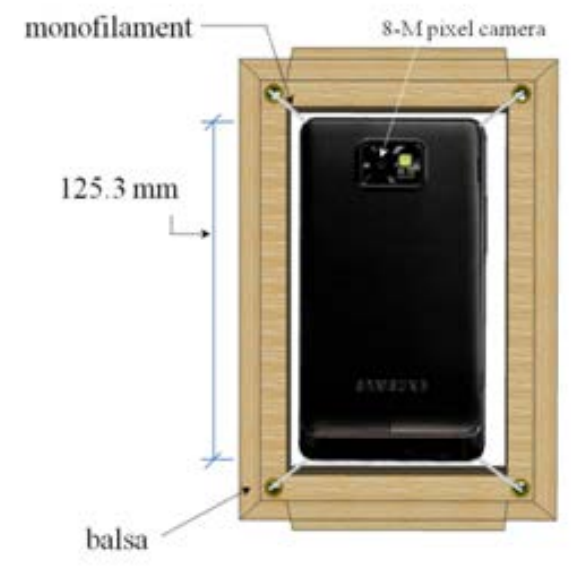

Figure 4. Vibration-proof device designed to reduce highfrequency vibrations in an electric glider. The smartphone is attached to the balsa framework with monofilament.

\subsection{Accuracy of Experiments}

\subsubsection{Position accuracy}

In the static and dynamic experiments, 3D location information, 3-axis attitude information and image were obtained from GPS built in smartphone, gyroscope and camera, respectively. The standard deviation in the location accuracy of GPS built in smartphone was $9.66 \mathrm{~m}$ for the static experiment and $13.81 \mathrm{~m}$ for the dynamic experiment. These results were similar to those from existing studies using MEMS sensors .

\subsubsection{Attitude accuracy}

Angular rate sensors have played an important role in aviation, space exploration and military applications. The heading angle provided by MEMS gyroscope in the static condition include the long-term bias drift, a slow-changing error source unlike the roll and pitch. The bias drift of the gyros is significant in MEMS-based systems and if left uncorrected can completely mask the navigation solution (Foxlin, 2005; Aggarwal et al., 2011).

\subsubsection{Image block triangulation}

The first dynamic experiment was conducted with smartphone directly attached to the bottom of the fixed wing of UAV without using separate vibration-proof device. In this experiment, there occurred contortion in the images taken by smartphones due to the high-frequency vibration directly delivered from UAV. In particular, such contortion occurred most frequently in the images obtained by Galaxy S. In addition, as for the ortho-images converted from the images with rare contortion among the images obtained from Galaxy S2 through triangulation, there was a negative consequence that the image point's residual RMSE was large.

Therefore, the image that best covers the test area for dynamic experiment was selected among those obtained from each smartphone. Using 12 images for Galaxy $\mathrm{S}$ and 8 images for both S2 type A and B, the image block was formed.

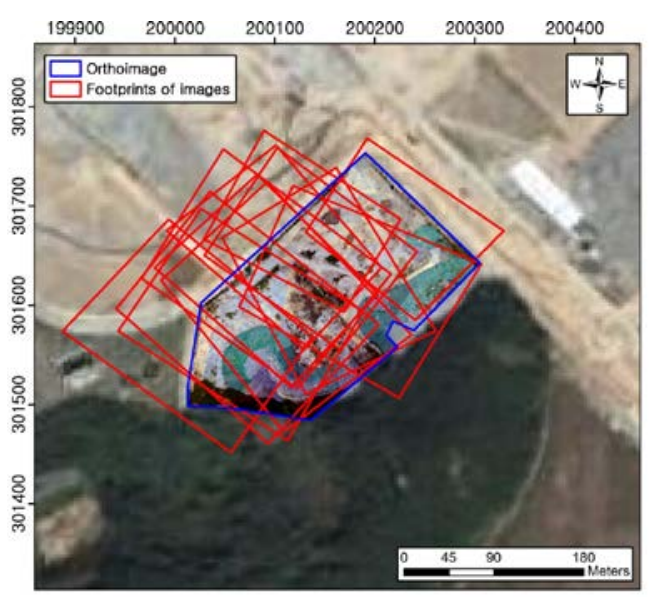

(a) 


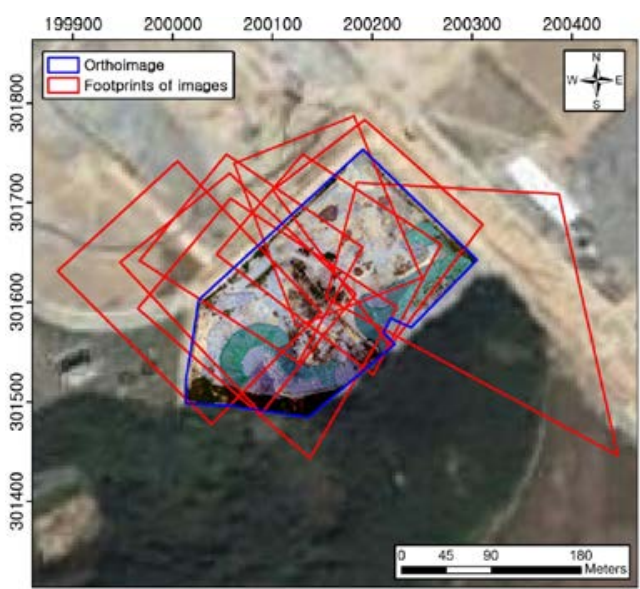

(b)

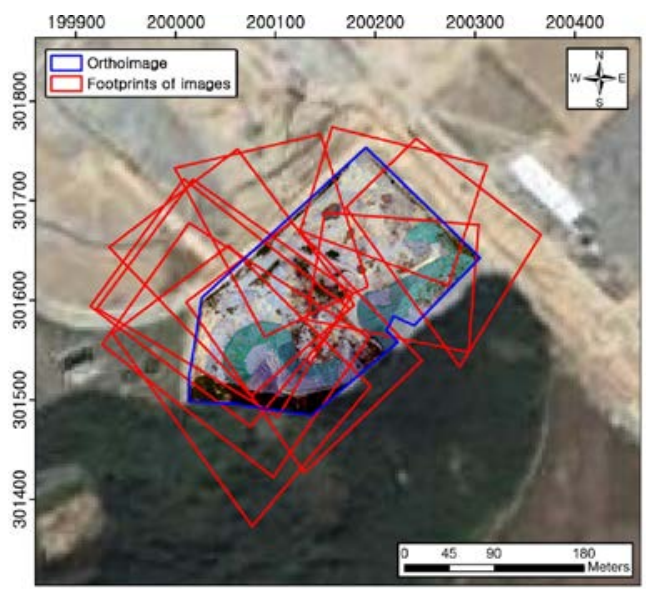

(c)

Figure 7. Overview of the image block: (a) Galaxy S, (b) S2 type A, and (c) S2 type B.

The image triangulation was conducted in accordance with presence or absence of consideration of the interior orientation (IO) parameters determined by camera calibration. In case IO parameters were taken into account in the static experiment, the results from triangulation for any smartphone type were within 1.5 pixel (RMSE), which was improved at least by 35\% compared to when IO parameters were not taken into account. On the contrary, the improvement effect of considering IO parameters on accuracy in triangulation for smartphone images in dynamic experiment was not significant compared to the static experiment. It was due to the significant impact of vibration and sudden attitude change of UAV on the actuator for automatic focus control within the camera built in smartphone under the dynamic condition.

\section{CONCLUSIONS}

This paper introduced a new photogrammetric UAV system using the smartphone technology. Although these results showed slightly lower accuracy than the results using existing system consisting of expensive sensors, they seems to be satisfactory in terms of cost and efficiency in that they were generated by only one smartphone. And this paper assessed the feasibility of smartphone as a payload for photogrammetric UAV system. Considering that these results were obtained from only 1 smartphone, they suggest that smartphone is not only very feasible as a payload for UAV system but also play direct or indirect roles loaded to UAV system. In addition, it is expected that the performances of MEMS sensors built in smartphone e will develop continuously, which in turn will increase the use of smartphone as a payload for photogrammetric UAV system in the future.

\section{REPERENCES}

Aggarwal, P., Thomas, D., Ojeda, L., Borenstein, J., 2011. Map matching and heuristic elimination of gyro drift for personal navigation systems in GPS-denied conditions. Measurement science and technology, 22, 1-12.

Bogacki, M., Malkowski, W., Misiewicz, K., 2008. Kite aerial photography (KAP) as a tool for completing GIS models. Ptolemais (Libya) case study. Lasaponara, R., Masini, N., Aracne(Eds.), Remote Sensing for Archaeology and Cultural Heritage Management. In: the 1st International EARSeL Workshop. CNR, Roma, 329-332.

Fotinopoulos, V., 2004. Balloon photogrammetry for archaeological surveys, In: International Archives of the Photogrammetry, Remote Sensing and Spatial Information Sciences, XX ISPRS Congress, Istanbul, Turkey, XXXV-B5, 504-507.

Foxlin, E., 2005, Pedestrian tracking with shoe-mounted inertial sensors. IEEE Computer Graphics and Application, 25, 38-46.

Mihajlovic, D., Mitrovic, M., Cvijetinovic, Z., Vojinovic, M., 2008. Photogrammetry of archaeological site Felix Romuliana at Gamzigrad using aerial digital camera and non-metric digital camera. In: ISPRS Archives Vol. XXXVII, ISPRS Congress, Beijing, China, pp. 397-399.

Przybilla, H.-J., Wester-Ebbinghaus, W., 1979. Bildflug mit ferngelenktem Kleinflugzeug. Bildmessung und Luftbildwesen, Zeitschrift fuer Photogrammetrie und Fernerkundung, 47, 137-142.

Remondino, F., Gruen, A., Von Schwerin, J., Eisenbeiss, H., Rizzi, A., Sauerbier, M., Richards-Rissetto, H., 2009: Multisensors 3D documentation of the Maya site of Copan. In: ISPRS Archives Vol. XXXVIII-3/W8, XXII CIPA Symposium, Kyoto, Japan.

Samsung mobile, 2012a. Samsung Galaxy S Specification, http://www.samsung.com/global/microsite/galaxys/specificatio n/spec.html (05 April 2012).

Samsung mobile, 2012b. Samsung Galaxy S2 Specification, http://www.samsung.com/global/microsite/galaxys2/html/speci fication.html (05 April 2012).

Wzorek, M., Landen, D., Doherty, P., 2006. GSM Technology as a Communication Media for an Autonomous Unmanned Aerial Vehicle. In: Proceedings of the 21st Bristol International Conference on UAV Systems. http://www.ida.liu.se/ davla/papers/gsm.pdf (10 March 2012).

\section{ACKNOWLEDGEMENTS}

This work was researched by the supporting project to educate GIS experts. 